\title{
Complicaciones asociadas a la trombocitopenia profunda en pacientes con dengue
}

\author{
Fredi Alexander Díaz-Q uijano ${ }^{1}$, \\ Luis Angel Villar-Centeno르, Ruth Aralí Martínez-Vega². \\ Complications associated to \\ severe thrombocytopenia \\ in patients with dengue
}

Background: Thrombocytopenia is a distinctive characteristic of dengue; however, few studies have related thrombocytopenia with its clinical severity. Aim: To identify the complications of dengue, associated to severe thrombocytopenia, defined as a platelets count below $50,000 / \mathrm{mm}^{3}$ ). Patients and Methods: Patients with clinical and serologic (positive IgM) diagnosis of dengue, consulting at hospitals located in Santander, Colombia, during the period 1993-1998, were studied. Clinical findings and laboratory tests (including hematocrit and platelets counts) were registered. The association between severe thrombocytopenia and the presence of complications, such as hemorrhagic manifestations (positive tourniquet test, petechiae, ecchymoses, bleeding from gums, epistaxis, hematemesis, hematuria and metrorrhagia) and signs of plasma leakage (pleural effusions and haemoconcentration), were evaluated using a univariate and multivariate analysis. Results: Of the 790 patients enrolled, 502 had a platelet count below $50,000 / \mathrm{mm}^{3}$. Severe thrombocytopenia was associated with hemorrhagic manifestations (OR=3.16; 95\% CI: 2.094.76; $\mathrm{p}<0.0001$ ) and signs of plasma leakage (OR=2.67; 95\% CI: 1.86-3.84; $\mathrm{p}<0.0001)$. The associations between severe thrombocytopenia and the complications of dengue (with exception of bleeding from gums, hematuria and metrorrhagia), were confirmed with the multivariated analysis ( $p$ <0.05). Conclusion: There was a strong association between deep thrombocytopenia and the severity of dengue (Rev Méd Chile 2006; 134: 167-73).

(Key words: Dengue hemorrhagic fever; Purpura, thrombocytopenic; Thrombocytopenia)

Recibido el 5 de mayo, 2005. Aceptado el 27 de julio, 2005.

${ }^{1}$ Centro de Investigaciones Epidemiológicas y ${ }^{2}$ Escuela de Medicina, Universidad Industrial de Santander, Bucaramanga, Colombia.

Correspondencia a: Dr. Fredi Alexander Díaz-Quijano. Centro de Investigaciones Epidemiológicas, Facultad de Salud UIS. Cra. 32 № 29-31 tercer piso. Bucaramanga, Colombia. Telefax: (7) 6345781. E-mail: fre_diazq@yahoo.com 
$\mathrm{E}^{1}$ dengue es la enfermedad producida por arbovirus más importante en el mundo, con alta incidencia en Asia, África, América Central y del Sur ${ }^{1}$. Se estima que anualmente ocurren entre 50 y 100 millones de infecciones por el virus del dengue, principalmente en las áreas tropicales y subtropicales infestadas por sus vectores, las especies de mosquito Aedes aegypti y Aedes albopictus $^{1,2}$. Durante la última década, en Sudamérica se ha registrado el más dramático incremento de la actividad del dengue, especialmente en Colombia, Ecuador, Paraguay, Perú, Venezuela y Brasil $^{2-7}$. Actualmente, en este último país se produce aproximadamente el $70 \%$ de los casos en las Américas ${ }^{3}$.

En Colombia, el dengue es endemo-epidémico, en zonas por debajo de los 1.800 metros sobre el nivel del mar y gran parte de nuestra población está a riesgo ${ }^{7-9}$. En el Departamento de Santander, al noreste del país, las tasas de incidencia se han incrementado en los últimos años, oscilando entre 113,4 y 268,7 casos por 100.000 habitantes ${ }^{8,9}$.

La mayoría de los individuos que adquieren la infección y enferman, finalmente evolucionan hacia dengue clásico (DC), enfermedad febril autolimitada usualmente sin complicaciones ${ }^{1}$. Sin embargo, una proporción variable de pacientes desarrolla hemorragias espontáneas, disminución del recuento de plaquetas y signos de extravasación del plasma. La conjunción de estas manifestaciones define el síndrome de dengue hemorrágico $(\mathrm{DH})^{1,10}$.

La intensidad de la trombocitopenia ha sido tomada como un parámetro para clasificar la severidad del dengue ${ }^{10}$. La OMS sugiere que para considerar un caso de $\mathrm{DH}$, éste debe presentar al menos un recuento de plaquetas inferior a 100.000/ $\mathrm{mm}^{3}$. Por otra parte, se recomienda como criterio de egreso hospitalario, la evolución hacia recuentos de plaquetas superiores a $50.000 / \mathrm{mm}^{3}$ (1).

A pesar de lo expuesto, existe escasa evidencia que respalde la asociación entre la intensidad de la trombocitopenia y las complicaciones de esta enfermedad $^{11-13}$. Paradójicamente, un estudio sugiere que podría no existir una clara relación entre el descenso de las plaquetas y la aparición de manifestaciones hemorrágicas ${ }^{13}$. El objetivo del presente estudio es determinar si existen y cuáles son las complicaciones asociadas a la trombocito- penia profunda $\left(<50.000\right.$ plaquetas $\left./ \mathrm{mm}^{3}\right)$ en pacientes con dengue.

\section{PACIENTES Y MÉTODOS}

Diseño. Estudio observacional analítico de corte transversal de pacientes hospitalizados en instituciones de Santander-Colombia, notificados a la Secretaría de Salud Departamental durante el período de octubre de 1993 hasta abril de 1998, con evidencia de infección aguda por virus del dengue.

Criterios de inclusión. Pacientes con síndrome febril agudo y manifestaciones compatibles con dengue, cuya información clínica permita clasificar la severidad de la enfermedad, lo que incluye al menos dos hematocritos en los pacientes sin efusiones pleurales.

Además, una prueba serológica (IgM) positiva (especificidad: 99,1\%; valor predictivo positivo en Santander: 96,2\%) ${ }^{14}$.

Evolución. Se consideraron como variables dependientes las manifestaciones hemorrágicas; la extravasación plasmática documentada por un viraje del hematocrito (Hto) $>20 \% 0$ evidencia de efusión pleural o ascitis; presencia de edema en miembros inferiores; y el síndrome de $\mathrm{DH}$, definido según los criterios de la OMS (plaquetas menores de 100.000/ $\mathrm{mm}^{3}$, extravasación plasmática y manifestaciones hemorrágicas) ${ }^{10}$. Los pacientes que no reunieron todos los criterios de $\mathrm{DH}$ se consideraron como casos de DC.

Selección y tamaño de la muestra. Se incluyó el total de la población de estudio.

Encuesta: La información clínica se obtuvo de una ficha de registro clínico-epidemiológico para dengue del departamento, aplicada en el momento de la toma de la muestra de sangre para el estudio serológico. En ella se incluyen síntomas, signos y pruebas de laboratorios presentes hasta el momento de la encuesta.

Laboratorio. En la atención inicial de urgencias y durante el periodo de hospitalización, se determi- 
naron los valores de Hto y recuento de plaquetas de cada día. En muestras de suero obtenidas después del quinto día de enfermedad, se realizaron pruebas serológicas de inmunoensayo enzimático para la detección de IgM específica para virus del dengue.

Estadística. La información demográfica, clínica y serológica de los pacientes, fue almacenada en una base de datos electrónica (Microsoft Excel 97, Microsoft Corp.). Las definiciones de los desenlaces fueron aplicadas en esta base de forma sistemática a todos los pacientes. Para evaluar la asociación de la trombocitopenia profunda (plaquetas $\leq 50.000 / \mathrm{mm}^{3}$ ) con los síntomas (cefalea, dolor retroocular, malestar, escalofrío, etc.) y las complicaciones (manifestaciones hemorrágicas, signos de extravasación plasmática, DH), se utilizó la prueba de Chi cuadrado $\left(\chi^{2}\right)$ determinando Odds Ratio (OR) con intervalos de confianza 95\% (IC 95\%). Posteriormente, para evaluar estas asociaciones, se realizó un análisis multivariado utilizando un modelo de regresión logística, que incluyó las variables de género, edad ( $<15$ años) y tiempo de evolución de la enfermedad al momento del ingreso ( $>4$ días). En el análisis de los datos se empleó el programa estadístico STATA versión 5.0 (Stata Corp.).

\section{RESULTADOS}

En total, se obtuvo información de 1.119 pacientes. Las pruebas de IgM específica para dengue se realizaron en 904 pacientes, siendo positiva en 891 (98,6\%). De ellos, sólo 790 pacientes tenían información clínica suficiente para clasificar la enfermedad (grupo incluido en el análisis).

En la población estudiada, la edad promedio fue 27 años (DE: 17,7; IC 95\%: 26-28,4) y la proporción de pacientes de género masculino fue de $55,8 \%$ ( $\mathrm{n}=441)$. Se documentaron 190 casos de DH $(24,1 \%)$, cuya edad fue similar a la de los demás pacientes, sin embargo, esta complicación estuvo significativamente asociada al género femenino (Tabla 1).

En general, el promedio del conteo mínimo de plaquetas fue $49,974 / \mathrm{mm}^{3}$ (DE: 39,253 ; IC 95\%: 47,232-52,715), siendo significativamente inferior en los casos de DH comparado con el de aquellos con DC (Tabla 1). Como punto de corte, el valor que mejor discriminó las poblaciones según su severidad, fue el recuento de plaquetas de $50.000 / \mathrm{mm}^{3}$, por debajo del cual se obtuvo la mayor asociación con DH (OR=5,4; IC 95\%: 3,49), con una alta sensibilidad $(87,4 \%)$ y una especificidad intermedia (44\%) para identificar este desenlace.

Tabla 1. Características de los pacientes con dengue, DC y D H

\begin{tabular}{|c|c|c|c|c|}
\hline \multirow[b]{2}{*}{ Características basales } & \multirow{2}{*}{$\begin{array}{c}\text { Total } \\
(\mathrm{n}=790)\end{array}$} & \multicolumn{2}{|c|}{ Según su severidad } & \multirow[b]{2}{*}{ Valor $\mathrm{p}$} \\
\hline & & $\mathrm{DC}(\mathrm{n}=600)$ & $\mathrm{DH}(\mathrm{n}=190)$ & \\
\hline Sexo masculino - No (\%) & $441(55,8)$ & $351(58,5)$ & $90(47,4)$ & 0,007 \\
\hline Edad (años) - Media $\pm \mathrm{DE}$ & $27,2 \pm 17,7$ & $27,3 \pm 17,4$ & $27 \pm 18,7$ & 0,83 \\
\hline \multicolumn{5}{|l|}{ Tiempo de evolución al ingreso } \\
\hline (días) - Media $\pm D E$ & $5 \pm 3,2$ & $4,8 \pm 2,9$ & $5,6 \pm 3,9$ & 0,002 \\
\hline$>4$ días de enfermedad - No (\%) & $439(55,6)$ & $322(53,7)$ & $117(61,6)$ & 0,06 \\
\hline \multicolumn{5}{|l|}{ Criterios de severidad: } \\
\hline Hemorraqias espontáneas - No (\%) & $629(79,7)$ & $452(75,5)$ & $177(93,2)$ & $<0,0001$ \\
\hline Prueba torniquete positiva - No (\%) & $287 / 602(47,7)$ & $218 / 476(45,8)$ & $69 / 126(54,8)$ & 0,07 \\
\hline Efusión pleural - No (\%) & $38 / 720(5,3)$ & $4 / 545(0,7)$ & $34 / 175(19,4)$ & $<0,0001$ \\
\hline Viraje del Hto >20\% - No (\%) & $189 / 780(24,2)$ & $25(4,2)$ & $164(89,6)$ & $<0,0001$ \\
\hline Conteo mínimo de plaquetas & & & & \\
\hline$\left(\mathrm{x} 10^{3} / \mathrm{mm}^{3}\right)-$ Media $\pm \mathrm{DE}$ & $50 \pm 39,3$ & $56 \pm 41,8$ & $30,8 \pm 20,1$ & $<0,0001$ \\
\hline
\end{tabular}




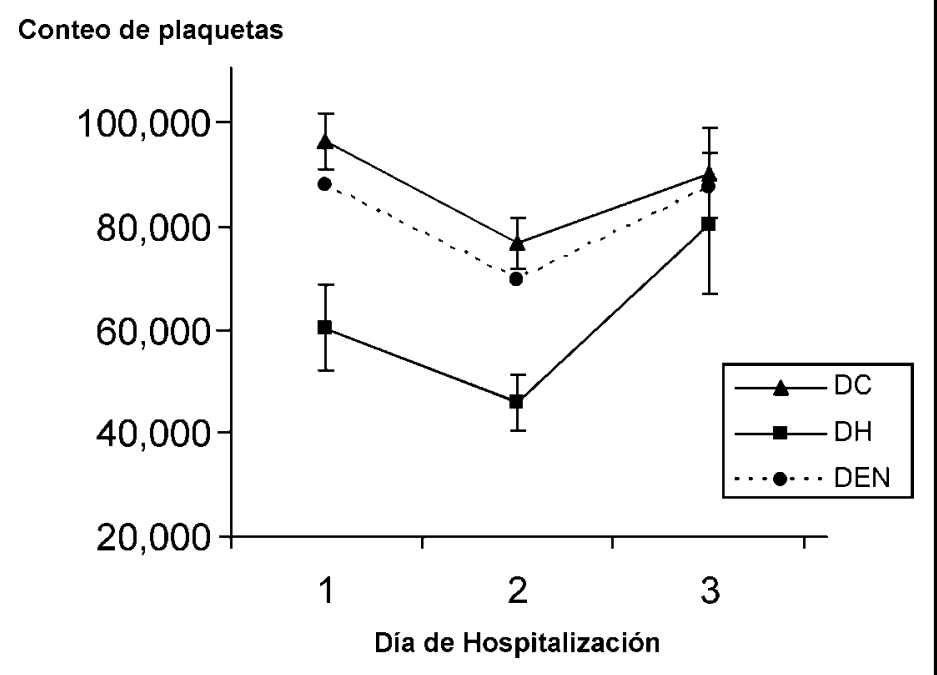

Figura 1. Se muestra el promedio de plaquetas en los primeros 3 días de hospitalización, en el total de pacientes con dengue (DEN) y en los subgrupos de DC y DH. Las barras de error representan el intervalo de confianza $95 \%$.

En general, el conteo de plaquetas más bajo se registró en el segundo día de hospitalización (Figura 1). Las diferencias entre DC y DH sólo se observaron en los dos primeros días ( $p<0,0001$ ). La trombocitopenia profunda, que se presentó en 502 pacientes, no estuvo asociada al sexo, pero fue más frecuente entre los pacientes mayores de 15 años (Tabla 2).

Con relación a los síntomas, en el análisis bivariado, la trombocitopenia profunda se encontró asociada a una mayor frecuencia de cefalea, malestar general, artralgias, dolor abdominal, vómito y diarrea. Estas asociaciones, con excepción de las observadas con cefalea y malestar general, fueron consistentes en el análisis multivariado
(Tabla 3). El dolor de garganta fue menos frecuente en los pacientes con trombocitopenia profunda, pero esta asociación no se sostuvo en el análisis multivariado.

Las manifestaciones hemorrágicas, incluyendo a las espontáneas y a la prueba del torniquete, fueron hallazgos más frecuentes en los pacientes con trombocitopenia profunda. En el análisis de regresión logística, estas asociaciones se mantienen para la prueba del torniquete y para las siguientes manifestaciones hemorrágicas espontáneas: petequias, equimosis, hematemesis y epistaxis (Tabla 4).

La extravasación plasmática, documentada principalmente por el viraje del hematocrito, se

Tabla 2. C aracterísticas de los pacientes con dengue según la intensidad de la trombocitopenia

\begin{tabular}{|lcccc|}
\hline Característica & \multicolumn{2}{c}{ Conteo de plaquetas } \\
& $\begin{array}{c}>0.000 / \mathrm{mm}^{3} \\
(\mathrm{n}=502)\end{array}$ & $\begin{array}{c}>50.000 / \mathrm{mm}^{3} \\
(\mathrm{n}=288)\end{array}$ & OR & IC $95 \%$ \\
\hline Sexo masculino - No (\%) & $283(56,4)$ & $158(54,9)$ & 1,06 & $0,79-1,42$ \\
Edad (años) - Media \pm DE & $29,5 \pm 17,8$ & $23,2 \pm 16,76$ & & \\
$\quad<15$ años - No (\%) & $112(22,3)$ & $111(38,5)$ & 0,46 & $0,33-0,63^{*}$ \\
Tiempo de evolución (días) - Media $\pm \mathrm{DE}$ & $4,8 \pm 3,2$ & $5,1 \pm 3,2$ & & 1,33 \\
$\quad>4$ días de enfermedad - No (\%) & $292(58,2)$ & $147(51)$ & $1,1,78^{*}$ \\
\hline
\end{tabular}

$*_{p}<0,0001$

$t_{\mathrm{p}}=0,05$ ?? 
presentó asociada a la trombocitopenia profunda. Consecuentemente, hubo una asociación estadísticamente significativa entre un recuento de plaquetas $\leq 50.000 / \mathrm{mm}^{3}$ y el síndrome de DH (Tabla 5).

\section{DisCUSIÓN}

La trombocitopenia es un rasgo distintivo del dengue y se ha descrito que su intensidad se correlaciona con el grado de viremia ${ }^{15}$ y con la magnitud de la respuesta inmune ${ }^{16-18}$. Se ha sugerido que, un descenso progresivo de las plaquetas puede anticipar la forma severa del dengue $^{19,20}$. Sin embargo, la evidencia disponible en la literatura que respalda estas aseveraciones es escasa $^{11-13}$.

Un análisis de 39 casos de adultos con dengue, mostró que las hemorragias mucocutáneas se acompañan de recuentos de plaquetas inferio-

Tabla 3. M anifestaciones clínicas asociadas a la trombocitopenia profunda

\begin{tabular}{|c|c|c|c|c|c|c|c|c|}
\hline \multirow[b]{2}{*}{ Síntomas } & \multicolumn{2}{|c|}{ Conteo de plaquetas } & \multicolumn{3}{|c|}{ Análisis bivariado } & \multicolumn{3}{|c|}{ Análisis multivariado } \\
\hline & $\begin{array}{c}\leq 50.000 / \mathrm{mm}^{3} \\
(\mathrm{n}=502)\end{array}$ & $\begin{array}{c}>50.000 / \mathrm{mm}^{3} \\
\quad(\mathrm{n}=288)\end{array}$ & OR & IC $95 \%$ & $\mathrm{p}$ & OR* & IC $95 \%$ & $\mathrm{p}$ \\
\hline Cefalea - No (\%) & $464(92)$ & $253(88)$ & 1,7 & $1,1-2,8$ & 0,03 & 1,5 & $0,9-2,5$ & 0,10 \\
\hline Malestar & 465 (93) & $253(88)$ & 1,7 & $1,1-2,8$ & 0,02 & 1,4 & $0,8-2,3$ & 0,24 \\
\hline Artralgias & $287(57)$ & $129(45)$ & 1,7 & $1,2-2,2$ & $<0,001$ & 1,5 & $1,1-2$ & 0,01 \\
\hline Dolor garganta & $125(25)$ & $91(32)$ & 0,7 & $0,5-1$ & 0,04 & 0,7 & $0,5-1$ & 0,06 \\
\hline Dolor abdominal & $76(15)$ & $20(7)$ & 2,4 & $1,4-4$ & $<0,001$ & 2,3 & $1,4-3,9$ & 0,002 \\
\hline Vómito & $407(81)$ & $211(73)$ & 1,6 & $1,1-2,2$ & 0,01 & 1,5 & $1,1-2,2$ & 0,02 \\
\hline Diarrea $^{\dagger}$ & $72(47)$ & $11(28)$ & 2,3 & $1,1-5$ & 0,03 & 2,3 & $1,1-4,9$ & 0,04 \\
\hline
\end{tabular}

*Ajustado por género, edad ( $<15$ años) y tiempo de enfermedad ( $>4$ días).

${ }^{\dagger} \mathrm{n}=193$ (153 vs 40)

Tabla 4. M anifestaciones hemorrágicas asociadas a la trombocitopenia profunda en pacientes con dengue

\begin{tabular}{|c|c|c|c|c|c|c|c|c|}
\hline \multirow[b]{2}{*}{$\begin{array}{l}\text { Manifestaciones } \\
\text { hemorrágicas }\end{array}$} & \multicolumn{2}{|c|}{ Conteo de plaquetas (por $\mathrm{mm}^{3}$ ) } & \multicolumn{3}{|c|}{ Análisis bivariado } & \multicolumn{3}{|c|}{ Análisis multivariado } \\
\hline & $\begin{array}{c}\leq 50.000 / \mathrm{mm}^{3} \\
\quad(\mathrm{n}=502)\end{array}$ & $\begin{array}{c}>50.000 / \mathrm{mm}^{3} \\
(\mathrm{n}=288)\end{array}$ & OR & IC 95\% & $\mathrm{p}$ & $\mathrm{OR}^{*}$ & IC 95\% & $\mathrm{p}$ \\
\hline Total - No (\%) & $458(91)$ & $221(76,7)$ & 3,2 & $2,1-4,8<$ & 0,0001 & 3,3 & $2,2-5,1<$ & 0,001 \\
\hline P. tomiquete ${ }^{\dagger}$ & $183(53)$ & $104(40)$ & 1,7 & $1,2-2,3$ & 0,002 & 1,8 & $1,3-2,5$ & 0,001 \\
\hline Espontáneas & $423(84)$ & $206(72)$ & 2,2 & $1,5-3,1<$ & 0,0001 & 2,2 & $1,5-3,1<$ & 0,001 \\
\hline Petequias & $319(64)$ & $143(50)$ & 1,8 & $1,3-2,4$ & 0,0001 & 1,8 & $1,3-2,4<$ & 0,001 \\
\hline Equimosis & $107(21)$ & $35(12)$ & 2 & $1,3-3$ & 0,001 & 2 & 1,3-3 & 0,002 \\
\hline Hematemesis & 75 (15) & $20(7)$ & 2,4 & $1,4-3,9$ & $<0,001$ & 2,7 & $1,6-4,5<$ & $=0,001$ \\
\hline Melenas & 58 (12) & $21(7)$ & 1,7 & $1-2,8$ & 0,054 & 1,7 & $1-2,8$ & 0,07 \\
\hline Gingivorragia & $111(22)$ & $43(15)$ & 1,6 & $1,1-2,4$ & 0,01 & 1,5 & $1-2,2$ & 0,052 \\
\hline Epistaxis & 118 (24) & 49 (17) & 1,5 & $1-2,2$ & 0,03 & 1,6 & $1,1-2,5$ & 0,02 \\
\hline Hematuria $^{\ddagger}$ & $41(11)$ & $14(6)$ & 2,1 & $1,1-3,9$ & 0,02 & 1,9 & $1-3,6$ & 0,051 \\
\hline Metrorragia ${ }^{\S}$ & 37 (17) & $12(9)$ & 2 & $1-4$ & $<0,05$ & 1,7 & $0,9-3,5$ & 0,13 \\
\hline
\end{tabular}

*Ajustado por género, edad (<15 años) y tiempo de enfermedad ( $>4$ días). ${ }^{\dagger} \mathrm{n}=602$ (344 vs 258 ). ${ }^{\ddagger} \mathrm{n}=631$ (377 vs 254). $\S_{n}=349$ (219 vs 130). 
Tabla 5. Evidencia de fuga plasmática asociada a trombocitopenia profunda

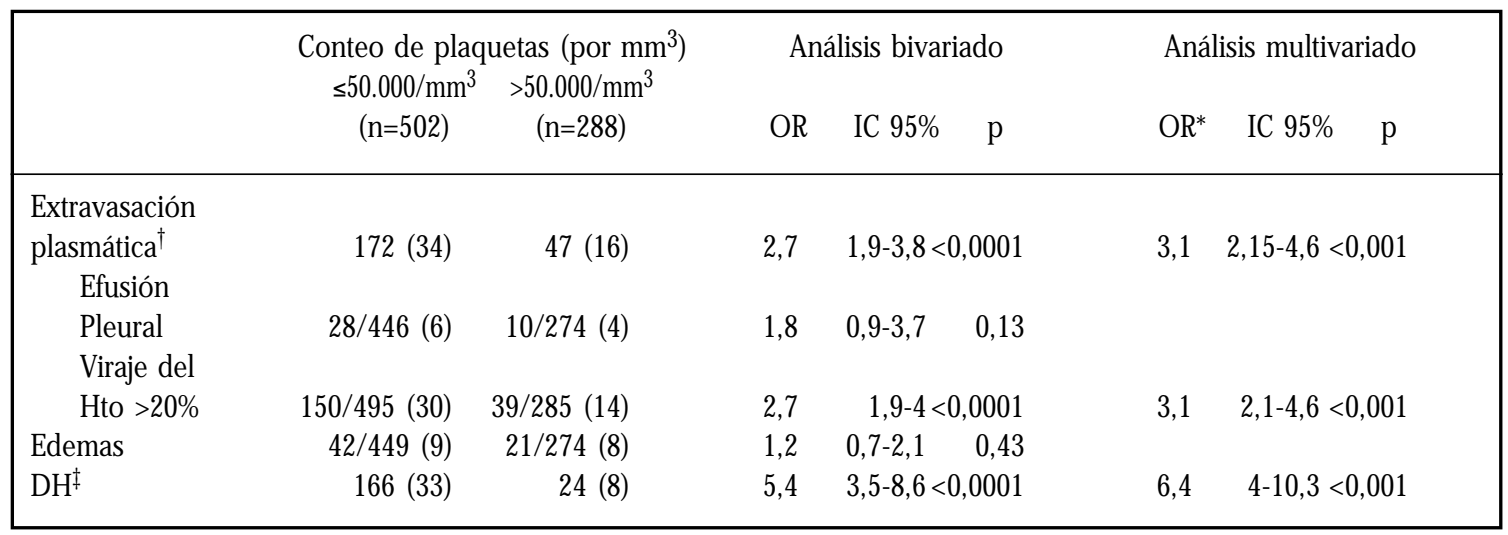

*Ajustado por género, edad ( $<15$ años) y tiempo de enfermedad ( $>4$ días). ${ }^{\dagger}$ Corresponde al criterio de la OMS: viraje del Hto $>20 \%$ o evidencia de efusión pleural o ascitis. 汭cluye evidencia de extravasación plasmática, un conteo de plaquetas $<100.000 / \mathrm{mm}^{3}$ y alguna manifestación hemorrágica.

res $^{12}$. Sin embargo, otro estudio, en 68 niños, no mostró asociación alguna entre el grado de trombocitopenia y la presencia de manifestaciones hemorrágicas ${ }^{13}$. No obstante, este último trabajo sí reveló una correlación entre el descenso de las plaquetas y la aparición de efusiones pleurales.

Los resultados del presente estudio sugieren que la presencia de trombocitopenia profunda se asocia tanto con las complicaciones hemorrágicas, como con la evidencia de extravasación plasmática, independientemente de variables como edad, género o el tiempo de enfermedad al momento de la consulta. Así, los pacientes con recuentos plaquetarios inferiores a $50.000 / \mathrm{mm}^{3}$, presentan con más frecuencia hemorragias mucocutáneas $\mathrm{y}$, con mayor relevancia clínica, efusiones pleurales y hemorragias mayores, complicaciones que se consideran criterios importantes para clasificar la severidad de la enfermedad $1,5,13$.

Las asociaciones que mostraron la extravasación plasmática y las manifestaciones hemorrágicas con los bajos recuentos de plaquetas, explican la correlación entre la trombocitopenia profunda y una mayor frecuencia de DH (Tablas 3 y 4). Estos resultados respaldan la utilización de los recuentos de plaquetas como indicadores de severidad en dengue, por lo que también podrían aplicarse en la medición del efecto de intervenciones terapéuticas.
Otro hallazgo de interés es el de la correlación entre algunos síntomas y la intensidad de la trombocitopenia. Se evidenció una consistente asociación de manifestaciones clínicas como las artralgias, el dolor abdominal, el vómito y la diarrea, con la presencia de bajos recuentos de plaquetas. La aplicación práctica de estos hallazgos radica en que la identificación temprana de los síntomas señalados, podría señalar a aquellos pacientes con mayor riesgo de complicaciones, aun antes de disponer de los resultados de laboratorio ${ }^{21}$.

No obstante, la principal limitación de este estudio, es que la recolección de la información se realizó de manera simultánea con la evaluación del desenlace y pudo estar sujeta a sesgos de información. Por lo anterior, consideramos que los presentes resultados deben ser validados, idealmente, en una cohorte prospectiva.

En conclusión, este estudio sugiere que la trombocitopenia profunda está fuertemente relacionada con la severidad del dengue, estimada esta última en términos de hemorragias (mayores $\mathrm{y}$ menores) y signos de extravasación plasmática. Además, se evidenció una mayor frecuencia de síntomas como cefalea, malestar general, artralgias, dolor abdominal, vómito y diarrea, en aquellos pacientes con recuentos de plaquetas más bajos. Como se mencionó, recomendamos que los presentes hallazgos se evalúen en un estudio de cohorte. 


\section{REFERENCIAS}

1. Pan American Health Organization. Dengue and dengue hemorrhagic fever in the Americas: guidelines for prevention and control. Washington, DC: PAHO. Sci Publ 1994; 548: 69-70.

2. Stephenson JR. The problem with dengue. Trans $R$ Soc Trop Med Hyg 2005; 99: 643-6.

3. Siqueira JB Jr, Martelu CM, Coelho Ge, Simplicio $\mathrm{AC}, \mathrm{HATCH} \mathrm{DL}$. Dengue and dengue hemorrhagic fever, Brazil, 1981-2002. Emerg Infect Dis 2005; 11: 48-53.

4. Uzcategui NY, Comach G, Camacho D, Salcedo M, Cabello de Quintana M, JimÉnez M et al. Molecular epidemiology of dengue virus type 3 in Venezuela. J Gen Virol 2003; 84 (Pt 6): 1569-75.

5. Méndez A, González G. Dengue haemorrhagic fever in children: ten years of clinical experience. Biomedica 2003; 23: 180-93.

6. TESH RB. Viral hemorrhagic fevers of South America. Biomedica 2002; 22: 287-95.

7. Camacho T, de la Hoz F, Cárdenas V, Sánchez C, de CALDERón L, Pérez L et al. Incomplete surveillance of a dengue-2 epidemic in Ibague, Colombia, 1995-1997. Biomedica 2004; 24: 174-82.

8. SIVIGILA. Comportamiento por regiones del dengue en el 2001. Boletín Epidemiológico Semanal. Semana epidemiológica № 2. Enero 6 a 12 de 2002. www.col.ops-oms.org/sivigila/2002/ BOLE02_02.htm

9. SIVIGILA. Enfermedades de notificación obligatoria. Informe primer semestre de 2003. Boletín Epidemiológico Semanal. Semana epidemiológica № 26. Junio 22-28 de 2003. www.col.opsoms.org/sivigila/2003/BOLE26_03.pdf

10. Organización Panamericana de ia Salud. Definiciones de casos. Dengue. Boletín Epidemiológico 2000; 21: 14-5.

11. Murgue B, Deparis $X$, Chungue E, Cassar O, Roche C. Dengue: an evaluation of dengue severity in French Polynesia based on an analysis of 403 laboratory-confirmed cases. Trop Med Int Health 1999; 4: 765-73.

12. Desruelies F, Lamaury I, Roudier $M$, Goursaud $R$, Mahe A, Castanet J et al. Cutaneo-mucous manifestations of dengue. Ann Dermatol Venereol 1997; 124: 237-41.

13. Krishnamurti C, Kalayanarooj S, Cutting MA, Peat RA, Rothwel SW, Reid TJ ET AL. Mechanisms of hemorrhage in dengue without circulatory collapse. Am J Trop Med Hyg 2001; 65: 840-7.

14. Díaz-Quijano FA, Martínez-Vega RA, Ocazionez RE, Viliar-Centeno LA. Evaluación de la prueba de IgM en suero agudo para el diagnóstico del dengue en un área endémica. Enferm Infecc Microbiol Clin 2006; 24: 90-2.

15. Libraty DH, Endy TP, Houng HS, Green S, KalayaNarooj S, Suntayakorn S ET al. Differing influences of virus burden and immune activation on disease severity in secondary dengue-3 virus infections. J Infect Dis 2002; 185: 1213-21.

16. Saito M, Oishi K, Inoue S, Dimanno EM, Alera MT, RoBles AM et AL. Association of increased plateletassociated immunoglobulins with thrombocytopenia and the severity of disease in secondary dengue virus infections. Clin Exp Immunol 2004; 138: 299-303.

17. Matondang AV, Widodo D, Zulkarnain I, Rengganis I, TRihandini I, InAda K et al. The correlation between thrombopoietin and platelet count in adult dengue viral infection patients. Acta Med Indones 2004; 36: 62-9.

18. Oishi K, Inoue S, Cinco MT, Dimaano EM, Aiera MT, ALFON JA ET AL. Correlation between increased platelet-associated IgG and thrombocytopenia in secondary dengue virus infections. J Med Virol 2003; 71: 259-64.

19. Isturiz $R$, Gubler D, Brea del CastiLo J. Dengue and Dengue Hemorrhagic Fever in Latin America and the Caribbean. Infect Dis Clin North Am 2000; 14: 121-40.

20. Kalayanarooj S, Vaughn DW, Nimmannitya S, Green S, Suntayakorn S, Kunentrasai N et al. Early clinical and laboratory indicators of acute dengue illness. J Infect Dis 1997; 176: 313-21.

21. Díaz-Quijano FA, Martínez-Vega RA, Viшar-Centeno LA. Indicadores tempranos de severidad en Dengue. Enferm Infecc Microbiol Clin; en prensa.

Agradecimientos

En la recolección de los datos se contó con el apoyo de la Oficina de Epidemiología del Servicio Seccional de Salud del Departamento. Además, agradecemos a los doctores Adolfo Mauricio Harker, Sandra Convers y Claudia Ximena Méndez, por su valiosa colaboración en la digitación de la información clínica. 